\title{
Gen. 3, 17; 5, 29; 8, 21.
}

Ein Beitrag zur Quellenkritik der Biblischen Urgeschichte von $\mathbf{K}$. Budde.

Wenn ich gerade die drei oben aufgeführten Stellen einer vergleichenden Betrachtung unterziehe, so folge ich darin dem Vorgang von Professor D. Ed. Riehm in der Recension meiner "Biblischen Urgeschichte" (Theol. Stud. u. Krit. 1885 , S. 780 f.). Zwar war mir, was ihm dazu Anlal's gab, keineswegs entgangen, vielmehr steht schon seit $5 \mathrm{Jahren}$ in meinem Handexemplar des Genesistextes bei den betreffenden Worten in 8,21 die Bemerkung:

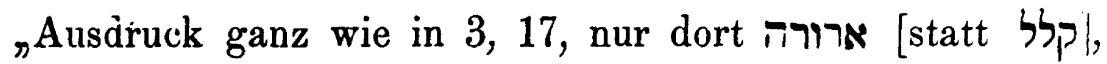
was keinen Unterschied macht. " Indessen hatte ich daraus doch keine weiteren Schlüsse gezogen, anders als aus dem Verhältnifs von 5, 29 zu 3, 17 : dafs dies auch hier möglich und geboten ist, wurde mir erst durch Riehm's Recension klar.

Indem Riehm die Beziehung der Weissagung Lamech's in 5, 29 auf den Weinbau Noah's entschieden verwirft, mifsbilligt er zugleich alle von Anderen vertretenen Auffassungen und trägt eine neue, eigene (vgl. schon Art. Noah in seinem Hdwrtrb. d. bibl. Alt. S. 1098 b) als ndie wahre Beziehung" vor. Lamech's Weissagung erfüllt sich ihm in 8, 21, in den Worten Jahwe's : „ich will den Erdboden nicht wieder um des Menschen willen verfluchen." Denn $\eta^{z w e i e r l e i ~ w i l l ~ J e h o v a ~ n a c h ~ 8, ~} 21$ infolge des Opfers Noahs, welches der Menschheit zugute kommt, nicht wieder thun : er will den Erdboden nicht wieder um des Menschen willen verfluchen, wie er nach Kap. 3, 17 gethan hatte, und er will nicht wieder alles, was da lebt, schlagen, wie er eben in der Sintfluth gethan hatte. Auf erstere segensvolle Wirkung der Opferdarbringung 
des gerechten Noah weist nun schon Gen. 5, 29 hin. Diese drei Stellen der jehovistischen Schrift 3, 17; 5, 29 und 8, 21 stehen wirklich in unlöslichem Zusammenhang. ${ }^{*}$ - Hat Riehm damit Recht, so fällt die Hauptstütze für die Annahme verschiedener Schichten in der jahvistischen Urgeschichte, da dann der Sündfluthbericht einen Abschnitt der ursprünglichen Erzählung bildet. Soll man das zugestehen, so mufs zu allererst der behauptete $\mathrm{Zu}$ sammenhang einen klaren, brauchbaren Sinn ergeben; den aber suche ich vergebens. Was heifst denn „den Erdboden nicht wieder um des Menschen willen verfluchen, wie er nach Kap. 3, 17 gethan hatte ${ }^{*}$ ? Da Riehm darüber keine Auskunft giebt, sondern sich an beiden Stellen auf die blofse Uebersetzung beschränkt, sind wir aufs Rathen angewiesen. Die nächstliegende Bedeutung wäre wohl, dafs Jahwe den in 3,17 ausgesprochenen Fluch nicht wiederholen, nicht erneuern will, ebenso wie der zweite Halbvers dies von der Sündfluth aussagt. Aber bedarf es denn solcher Erneuerung, wirkt nicht der einmal ausgesprochene Fluch von selbst weiter fort? Welche Wohlthat könnte also nach 8, 21 und 5, 29 darin liegen, dals er nicht wiederholt werden soll? Kann dies unmöglich die Meinung sein, so wollen wir einmal annehmen, es bedürfte zu dauernder Wirksamkeit dieses Fluches allerdings einer Erneuerung, sodals mit Unterlassung derselben der Fluch von 3, 17 aufser Kraft träte und dem Menschen eine Erleichterung geboten wäre - was müfsten wir dann in den Kauf nehmen! Zuerst bliebe uns der Verfasser den Aufschlufs darüber schuldig, wie lange die Wirkung des ersten Fluches gedauert hätte und in welchen Zwischenräumen eine Erneuerung nöthig wäre. Aber auch davon wollen wir absehen und annehmen, die Wendung stehe füglich für eine Aufhebung des Fluches, obgleich die schwerlich so ausgedrückt werden könnte. In jedem dieser Fälle wäre der in 3, 17 über den Erdboden ausgesprochene 
Fluch seit Noah's Zeit nicht mehr wirksam, damit auch seine Folge für den Menschen aufgehoben. Die Erde trüge seit der Zeit nicht mehr Dornen und Disteln, sondern wie vor dem Sündenfall lauter schöne Früchte; der Mensch brauchte nicht mehr das Kraut des Feldes zu essen, nicht mehr im Schweifse seines Angesichts zu arbeiten : sein Leben wäre ein Leben im Paradiese mit alleiniger Ausnahme der Sterblichkeit und - der Geburtsschmerzen des Weibes, das unerklärlicher Weise seinen besonderen Fluch behalten mufs, während der Mann des seinigen entledigt ist. Die Dogmatik aller Zeiten, ja das Christenthum als solches würde sich eine erhebliche Berichtigung nach diesem neuen and vermeintlich einzig richtigen Verständnils von Gen. 8, 21 zuziehen, und das allgemein menschliche, auf die Erfahrung begründete $\mathrm{Be}$ wufstsein würde sich belehren lassen müssen, dafs der Mensch kein Leben der Sorge, Mühe und Arbeit führt, dafs das goldene Zeitalter nicht im grauen Alterthum liegt, sondern schon seit der Sündfluth die Gegenwart der Menschen begleitet. - Die „Grundschrift" liefert den unwiderleglichen Beweis, dafs ein Hebräer eine Urgeschichte schreiben konnte, ohne ausdrücklich zu berichten, seit wann und warum das Menschenleben eitel Mühe und Arbeit ist; aber dafs jemand einen Bericht wie Gen. 2. 3 schreiben konnte, welcher so augenscheinlich die eigene Empfindung spiegelt, um dann die Thatsache selbst schon von Beginn der zweiten Menschheit an zu leugnen, das ist platterdings unmöglich. Unter diesen Umständen kann ich auch vorläufig nicht glauben, dals Riehm diese Auffassung vertreten will, und mufs von neuem fragen, welche andere Auffassung noch denkbar wäre.

Das לא אסיף לקלל עור אח הארמה kann an sich auch heifsen : „Ich will dem alten Fluch über den Erdboden keinen neuen hinzufügen ${ }^{*}$, mit anderen Worten „den auf dem Erdboden lastenden Fluch nicht noch verschärfen. ${ }^{*}$ 
Das würde 3,17 gegenüber nicht zu Ungereimtheiten führen, ja, das Beispiel einer solchen Verschärfung liegt, freilich in Gestalt eines Fluches über den Menschen, in 4, $11 \mathrm{f}$. vor. Aber wie Riehm's eigene Uebersetzung diese Auffassung kaum offen lälst, so spricht auch der hebräische Wortlaut und der Zusammenhang entschieden dagegen. Derselbe Ausdruck לא אסיף עור ל' bezeichnet in dem folgenden Halbverse nicht eine Steigerung oder Vermehrung, sondern eine Wiederholung des schon einmal Geschehenen und nun Vergangenen. Das בעבור הארם in 21 a steht nothwendig für $21 \mathrm{~b}$ mit, denn um die Sündfluth handelt es sich doch in erster Linie, und gerade in ihr hat die übrige Schöpfung nach Auffassung des Jahwisten in 6, 5-7 (anders die Grundschrift, vgl. meine „Bibl. Urgesch.“ S. 252 f.) um des Menschen willen unschuldig mitleiden müssen. Diese dort nothwendige Bedeutung des בעבור האדם in 3,17 . In der Sündfluth ist das Mitleiden der übrigen Schöpfung reines Accidens : was den Menschen vernichtet, ist die Fluth; ob sie gleichzeitig den Erdboden schädigt und die Thiere vernichtet, kann ihm gleichgültig sein. In 3,17 ist es gerade der Fluch über die Erde, durch welchen der Mensch getroffen wird, das „um deinetwillen " birgt einen starken finalen Beigeschmack. Der Unterschied ist nicht grofs, bedingt aber bei so enger Verschlingung der Sätze, wie sie in 8,21 vorliegt, ein Schielen des Gedankens, und auch darum ist es höchst unwahrscheinlich, dafs v. 21a auf 3, 17 zurückweisen sollte. Auch v. 22 verlangt einheitliche Beziehung, wie. sie durch den zur Hand liegenden Gegensatz der Sündfluth wirklich geboten wird : durch gleichzeitige Beziehung auf eine nur gedachte, in keiner Weise vorstellbar gemachte Verfluchung des Erdbodens entstände nur Unklarheit. Aber noch mehr. $\mathrm{Zu} 21 \mathrm{~b}$ allein, zu der Aussage, dafs Jahwe nicht wieder alles Lebendige schlagen wolle, enthält v. 22 mit seiner GeZeitschrift f. d. alttest. Wiss. Jahrgang 6. 1886. 
währleistung der Gesetze des Naturlauf gar nicht den entsprechenden Gegensatz, der doch darin liegen mufs. Und eben so wenig bietet $21 \mathrm{~b}$ ein ausreichendes Bild der Sündfluth, am wenigsten für den Jahwisten, der in seinem ' 7, 4. 23 mit vollem Bedacht die Pflanzen einschliefst und in 7, 22 die Thiere als Theil dieses Ganzen erwähnt; der das Wiedererwachen des Pflanzenwuchses nach der Sündfluth in dem frischen Oelblatt 8, 11 nicht vergifst. Es liegt auf der Hand, dals v. 21 a mit dem Verfluchen des Erdbodens und $21 \mathrm{~b}$ mit der Vernichtung der Lebewesen, wozwischen die doppelte Begründung durch בעבור uür beide Sätze gemeinschaftlich sich einschiebt, zusammenzuschliefsen sind zu einem einzigen Gesammtbilde, dem der Sündfluth, um die es sich hier allein handelt. Eben diese Einschiebung erklärt zugleich, warum in 21 b nicht mit einem blofsen ולהכות fortgefahren, sondern ein neuer, selbständiger Satz gebildet wird. Die Mehrzahl dieser Einwendungen gilt nicht nur gegen das letzterwähnte Verständnifs, sondern schliefst jede Beziehung von 8,21 a auf 3,17 aus. Immerhin wird es nützlich sein, diese letzte Auffassung noch an 5, 29 zu prüfen. Gott hat den Erdboden verflucht, und kraft dieses Fluches hat der Mensch Arbeit und Mühsal sein ganzes Leben lang. Diesen Zustand lälst Jahwe nach der Sündfluth bestehen, nimmt sich aber nach Noah's Opfer vor, ihn nicht durch einen weiteren Fluch über die Erde noch zu verschärfen. Und darauf sollte Lamech's Weissagung von Noah gehen : „Dieser wird uns trösten über unsere Arbeit und die Mühsal unserer Hände [wie sie hervorgeht] aus dem Erdboden, welchen Jahwe verflucht hat" (so etwa nach Riehm's Auffassung). Darin also bestände der Trost, dafs es nicht schlimmer wird, ein Seitenstück zu dem, was der Franzose bonheur allemand nennt, oder auch zu dem Gebet der Witwe, von dem Chamisso nach Luther erzählt. - So sind wir denn wieder bei dem Unmöglichen angelangt, 
und ich mufs daraus wiederum rückwärts schliefsen, dafs Riehm auch diese Auffassung nicht gemeint hat. Eine weitere Möglichkeit sehe ich aber nicht mehr, und bis Riehm eine solche nachgewiesen hat, muls ich annehmen, dals er sich durch den allgemeinen Schein einer günstigen Aeufserung mit Bezug auf 3, 17 hat blenden und genügen lassen, einen Schein, der sich bei näherem Zusehen nicht bestätigt.

Inzwischen möchte ich noch auf einiges hinweisen, was jeder Beziehung von 5, 29 auf 8, 21 im Wege steht. Das „dieser wird uns trösten" verlangt eine That Noah's, ein Handeln, das von ihm ausgeht. Statt dessen bietet 8, 21 einen Ausspruch Jahwe's, welcher einen Vorsatz für die Zukunft enthält und nur im allgemeinsten Zusammenhang steht mit dem Opfer, das Noah ohne irgend welche Abzweckung auf ein bestimmtes Ziel, dem Sinne gemäfs gewifs vor allem zum Dank für die erfahrene Gnade, dargebracht hat. Und mehr noch. Zwar bleibt das Opfer nicht ohne eine gewisse besänftigende Einwirkung auf Jahwe (wie das ריח הניחה beweist), aber der eigentliche Grund, weshalb er in Zukunft von solchen die ganze Welt in Mitleidenschaft ziehenden Strafgerichten absehen will, wird ausdrücklich angegeben, nämlich der, dafs es ja doch nichts hilft: „denn das Dichten des menschlichen Herzens ist böse von seiner Jugend an. " Hätte der Verfasser, wie Riehm zu 5, 29 annimmt, den Noah an dieser Stelle als Wohlthäter der Menschheit kennzeichnen wollen, er würde gewifs einen anderen Grund für Gottes Langmuth angegeben haben. Aber so darf ich mich nicht ausdrücken, wenn ich nicht wieder von Riehm dahin mifsverstanden werden will, als wenn ich die Erzähler der Genesis für Erdichter willkürlicher Märchen hielte (a. a. O. S. 778 Z. 11 v. u.). Ich sage daher ohne eine unmögliche Bedingung rein assertorisch : Da Jahwe einen so rücksichtslos demüthigenden Grund seiner Langmuth angiebt, so ist 
es dem Erzähler nicht eingefallen, hier Noah als Wohlthäter der Menschheit darzustellen oder ihn in $5,29 \mathrm{im}$ Ausblick auf diese Stelle als solchen verkünden zu lassen. Damit fällt aber zugleich die Beziehung von 8, 21 a auf 3, 17; denn niemand wird anders als dem Verständnifs von 5, 29 zuliebe sich versucht fühlen, die Stelle aus ihrer natürlichen Beziehung auf die Bündfluth herauszureifsen.

So mufs ich denn auf Kiehm's Belehrung in diesem Punkte verzichten und für 5, 29 bei der allen Ansprüchen genügenden Beziehung auf Noah's Weinbau vorläufig bleiben, obgleich damit - wie Riehm mit Bezug auf andere Stellen mir gegenüber zweimal nachdrücklich rügt die Stelle 5, 29 „alles religiös-sittlichen Inhalts bar ${ }^{\star}$ bleibt. Als Ausleger der heiligen Schrift bin ich zu sehr gewohnt auf den Pulsschlag des Textes selbst zu horchen und ihm sein Recht ungeschmälert zu gönnen, um mich von an sich und an der rechten Stelle löblichen erbaulichen Voraussetzungen und Wünschen auf seine Kosten leiten zu lassen ${ }^{1}$ ).

Nichtsdestoweniger bleibt wahr, was ich eingangs be-

1) Auch die Verweisung auf das ursprüngliche Nomadenthum Israel's und seine Abneigung gegen den Weinbau, auf Nasiräer und Rechabiten kann hier nichts verschlagen, weil eben das Stück 9, 20 -27 nicht den Standpunkt dieser Minderheit des geschichtlichen Israel, sondern den von Riehm selbst (Hdwrtrb. Art. „Wein" S. 1750 b) gezeichneten der Mehrheit vertritt. Sefshaftigkeit ist unbedingte Voraussetzung der in Fluch und Segen c. 9, 25-27 festgelegten Völkerverhältnisse, der Weinbau wird augenscheinlich als Kulturfortschritt gefafst und als solcher willkommen geheifsen. Nicht der verfluchte Sohn Kanaan hat den Wein gebaut oder gar sich daran berauscht, sondern der des Segens und Fluches mächtige Patriarch, und dafs jener den Berauschten verhöhnt hat, ist sein Vergehen. Der Rausch Noah's dagegen findet keine sittlich-religiöse Beurtheilung und ist gewifs als überraschende Wirkung des ersten Genusses von der noch unbekannten Gabe gedacht. Die ausnahmsweis hohe Werthung des Weines aber, die in 5, 29 hervortritt, stammt mir, wie auch Riehm S. 777 referirt, ron einem Späteren, dem Redactor $\mathrm{J}^{3}$. 
tonte, dafs ich Riehm für eine fruchtbare Anregung Dank schulde. So sicher 8, 21 a nicht auf 3,17 zurückweisen will, so wenig kann doch auch die auffallende Uebereinstimmung im Ausdruck als zufällig angesehen werden. Richtig hebt Riehm hervor, dal's von „Verfluchen des Erdbodens" in der ganzen Sündfluthgeschichte nicht die Rede ist - eben so wenig übrigens irgendwo sonst aufser unseren drei Stellen (zu vgl. Hiob 24, 18. 5, 3). Aber viel mehr bedeutet der Umstand, dafs die Wendung in der Sündfluthgeschichte auch sehr wenig angebracht ist. Unter einem Fluche versteht man doch eine geistige Macht, die Wesen und Lebensbedingungen des Betroffenen dauernd beeinflufst, vorwiegend gleichsam als zehrende Krankheit wirkt, jedenfalls aber, wo sie nicht widerrufen und aufgehoben wird, nicht anders zur Ruhe kommen kann als durch die Vernichtung des Betroffenen. Das alles trifft bei der Sündfluth nicht zu. Sie ist ein einzelnes, durchaus concretes Unheil, das die Erde trifft, das weder mit ihrer Vernichtung endet, noch auch - wie der Fluch in 3, 17 - dauernd einen schädigenden Einflufs auf sie ausübt. Denn nirgends wird davon etwas erwähnt. Die unveränderte Fortentwicklung des Pflanzenwuchses wird in 8, 11 als selbstverständlich vorausgesetzt, sobald das schwindende Wasser den Pflanzen, einer nach der anderen, ihre Lebensbedingungen wieder freigiebt; der Fortbestand der Menschen- und Thierwelt ist sorgfältig gesichert. Wird nun bei der Verhängung der Sündfluth von einem Fluche nichts gesagt, widerspricht der ganze Hergang diesem Begriffe, so muls die Wahl dieses Ausdrucks in dem Rückblick 8, 21 billig überraschen, und wir haben allen Grund, die Erklärung dafür an der einzigen selbständigen Stelle zu suchen, wo derselbe Ausdruck, und zwar dort als der denkbar angemessenste sich findet, nämlich in 3,17. Einheit des Verfassers der beiden Stellen erscheint sofort als ausgeschlossen, weil derselbe Verfasser den Ausdruck nicht in so ver- 
schiedenem, zu Mifsverständnifs verleitendem Sinne gebrauchen würde. So bietet der Vergleich der beiden Stellen cinen neuen Beweis, dafs der Verfasser des jahwistischen Sündfluthberichts von dem der Paradiesesgeschichte zu unterscheiden ist, mit anderen Worten, dafs die Paradiesesgeschichte nicht erst $\mathrm{zu} \mathrm{J} \mathrm{J}^{2}$, sondern schon $\mathrm{zu} \mathrm{J}^{1}$ gehört (vgl. Bibl. Urgesch. S. 230 ff.). Vielmehr wird die Wahl des sonderbaren Ausdrucks in 8, 21 eben daraus zu erklären sein, dafs der Verf. der Sündfluthgeschichte den Ausdruck fertig geprägt an jener Stelle vorfand. Was seinen Blick auf jene Stelle lenkte, wird in erster Linie das „um deinetwillen" gewesen sein, welches in so überraschender Weise zu dem unschuldigen Mitleiden des Erdbodens in der Sündfluth stimmte. Damit ist also ferner ein Punkt gewonnen, in dem die Abhängigkeit des $\mathrm{J}^{2}$ von $\mathrm{J}^{1}$ nicht nur im Stoff, wie in den Namen der Stammtafeln, den Namen Nimrod und Noah, sondern auch im Sprachgut hervortritt, ein willkommenes Seitenstück zu dem גב in 10, 8, vgl. mit demselben Worte in 10, 9 aus $\mathrm{J}^{1}$ (vgl. Bibl. Urgesch. S. 390 ff.). Beide Male ist das Wort beibehalten, die Bedeutung aber eine wesentlich andere geworden. Wie aber dort geschlossen werden mulste, dals der neue אבר Nimrod, der נבר בארץ, von $\mathrm{J}^{2}$ an die Stelle des alten צ' gesetzt, nicht dieser neben jenem beibehalten wurde, so darf endlich auch hier mit grofser Wahrscheinlichkeit geschlossen werden, dafs $\mathrm{J}^{2}$ durch den neuen Gebrauch des pל אל אח הארמה בעבור ראדם den alten, der sein Vorbild gewesen, ersetzte und beseitigte. Als denkender Schriftsteller, was er zweifellos war, wird er nicht so verschiedenen Gebrauch einer so hervorstechenden Formel in seinem zusammenhängenden Werke gelitten haben. Das aber bedeutet nichts Geringeres, als dals die Paradiesesgeschichte bei $J^{2}$ keine Aufnahme gefunden, da؟s er sie demnach durch eine andere Schöpfungsgeschichte ersetzt hat. Was ich erst am Schlusse meiner „Biblischen Urgeschichte" aus einem 
sehr verwickelten Indicienbeweis, ohne directe Zeugnisse zu erschliefsen wagen durfte (S. 470-495); was Riehm als der Gipfelpunkt aller Willkür und abenteuernden Kritik erscheint, sodal's er nur einen halb entrüsteten, halb höhnenden Ausruf, aber keine Widerlegung dafür übrig hat (S. 784) : dafür findet sich hier ein greifbares bestätigendes Zeugnifs. Und nun erst versteht man die Herübernahme jenes Ausdrucks in die Sündfluthgeschichte vollkommen. Schon lange vor der Entscheidung jener Frage hatte ich bemerkt (Bibl. Urgesch. S. 231), dafs „die Sündfluthgeschichte möglicherweise als Ersatz der Sündenfallsgeschichte gelten konnte, da sie ein Einreifsen und Wachsen des sündlichen Verderbens voraussetzt und von schwerer Strafe erzählt ${ }^{\alpha}$ : das scheint wirklich die Meinung von $\mathbf{J}^{2}$ gewesen zu sein. Ich will mich deutlicher ausdrücken. Als dieser Schriftsteller sich entschlofs die alten Berichte seines Volkes über die Urzeit durch ihm noch wahrer und werthvoller erscheinende zu ersetzen, wurde ihm dies wohl sehr erleichtert durch den Eindruck, dafs das Wesentlichste nicht verloren ginge, da sein Bericht über die Sündfluth den über den Sündenfall gewissermafsen in sich zu schliefsen schien. So ist es denn begreiflich, dafs für einen einzelnen Punkt, in welchem sich die neue, stellvertretende Erzählung auch stofflich mit der verdrängten älteren berührte, der scharf geprägte Ausdruck der letzteren beibehalten wurde, obgleich er an seine neue Stelle viel weniger pafste.

So scheint mir in dieser kleinen Einzelnheit aus der biblischen Urgeschichte eine nicht unwichtige Bestätigung zu liegen für diejenige Darstellung ihrer Entstehungsgeschichte, die ich, in manchen Punkten nach dem Vorgange Anderer, in dem öfter genannten Buche gegeben habe, eine Einzelheit, die einen besonders tiefen Einblick gerade in die verwickeltsten Fragen jener Geschichte gewährt. Auf dem Wege zu diesem Ergebnifs mulste ich die falschen Schlüsse Riehm's abwehren, und es war mir nicht unwill- 
kommen, mich dadurch einmal mit dieser rücksichtslos absprechenden, ausgesprochener Mafsen zum warnenden Beispiel auch für Andere bestimmten Recension auseinanderzusetzen. Indessen habe ich den hier besprochenen Punkt nicht deshalb aus vielen herausgegriffen, weil er mir zur Kennzeichnung jener Recension als der geeignetste erschienen wäre, sondern weil ich nicht mit einer blofsen Abwehr vor den Leser treten mochte, hier dagegen ein neues, positives Ergebnifs bieten konnte. Anhangsweise aber mag es mir gestattet sein, die allgemeineren Vorwürfe Riehm's, die Methode meiner Arbeit betreffend, zu berühren. Dieselben gelten theils mir allein, theils einer ganzen Reihe heutiger Forscher. Mir allein gilt aufser einer langen Vorhaltung, die Riehm zuletzt selbst als auf mein Buch nicht zutreffend zurückzieht (S. 756 f.), eine sehr scharfe Zurechtweisung über den Ausgangspunkt meiner Untersuchung. Zuerst allgemein gehalten (S. $757 \mathrm{f}$.) wird sie bestimmter (S. 770) folgendermafsen zusammen-

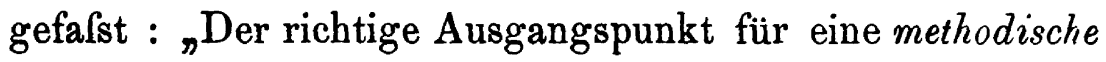
Untersuchung . . . . wäre . . . Gen. 4 gewesen. Dann hätten nicht Einfälle und gewaltsame Herstellungen eines angeblich ursprünglichen Textes die Untersuchung über dieses Kapitel beeinflufst." Der letzte Satz kann sich nur auf Nr. I und II meines Buches, über die Engelehen in $6,1-4$ und über den Baum des Lebens, beziehen. Wer indessen mein Buch aufmerksam liest, wird sich leicht überzeugen, dafs die Ergebnisse dieser Untersuchungen auf die vier folgenden nicht den allergeringsten Einflufs ausüben, sondern erst am Ende der siebenten wieder in Betracht kommen. Eben darum wurden sie vorausgeschickt, weil sie für sich ein unabhängiges Ganze bilden, sodals ich sie weder trennen, noch in den geschlossenen Gang der grofsen Untersuchung, welche erst mit III (Die Sethitentafel der Grundschrift) beginnt, störend einschieben durfte. Hätte Riehm die Worte „im wesentlichen" in 
meinem Vorwort, die er S. 757 abdruckt, auch berücksichtigt, so wäre ihm dieser Anstols vielleicht gehoben gewesen. Dafs ich aber in Unters. III nicht mit Gen. 4, sondern mit Gen. 5 beginne, geschieht darum, weil mein Buch nicht, wie Riehm S. 770 (ähnlich auch S. 756) angibt, untersuchen will,, ob in der jehovistischen Schrift schon verschiedene Schichten von einem Redactor miteinander verbunden waren", sondern sich laut Vorwort die umfassendere Aufgabe stellt, das Verhältnils der Quellen überhaupt, d. i. aller Quellen zu einander klar zu legen. Dafür war der gegebene Ausgangspunkt nicht Gen. 4, sondern Gen. 4. 5, d. h. die vorsündfluthlichen Stammtafeln, und mit gutem Bedacht habe ich mit der vollständigsten und besterhaltenen in Gen. 5 begonnen, um dann drei ganze Untersuchungen dem vierten Kapitel zu widmen. Was würde wohl Riehm dazu sagen, wenn man ihm erst ein anderes Ziel seiner Untersuchungen aufnöthigen wollte, um ihn dann über die befolgte Methode zu belehren!?

Gegen einen weiteren Kreis, mich eingeschlossen, richtet sich der Eingangsabschnitt der Recension S. 753 -755. Indem Riehm dabei an ein von ihm für möglich gehaltenes, gewifs irriges Verständnifs eines Ausspruchs von B. Stade in dieser Zeitschrift (1883, S. 2 Anm. 2) anknüpft, entwirft er nicht ohne einen gewissen Humor ein drastisches Bild des heutigen kritischen Verfahrens, wie es sich durch seine Brille darstellt, ein Bild, das augenscheinlich bestimmt ist, nach allen seinen Zügen durch den nachfolgenden Bericht über meine Biblische Urgeschichte seine Begründung zu finden. Riehm wird es mir nicht verargen, wenn ich mich in dem „Baumeister ${ }^{4}$ seiner Schilderung nicht wiedererkenne. Meine Methode habe ich wesentlich an den Werken der von ihm gepriesenen Meister geschult und glaube auf ihren Schultern zu stehen. Die harmonistisch-erbauliche Methode aber, zu der mir Riehm in dem oben beleuchteten Beispiel und anderwärts 
in seiner Recension nur gar zu sehr zu neigen scheint, habe ich weder bei de Wette noch bei Ewald, weder bei Hupfeld noch bei Bleek gefunden. Und heifst das in die Fufstapfen jener Männer treten, wenn man ängstlich bei ihren Ergebnissen stehen bleibt und sich durch die Furcht vor „dem Hohne der Feinde aller Kritik“ (S. 784) von weitergehenden Forschungen abhalten lälst? Oder ist es nicht gerade das Verdienst jenes früheren Geschlechtes, dafs wir auf festen Grundmauern weiterarbeiten dürfen, daf's wir eine durch sie gewonnene und geschulte Zuhörerschaft haben und nicht in jeder neuen wissenschaftlichen Arbeit $^{1}$ ) von neuem die Elemente apologetisch tractiren müssen. Wer das immer wieder meint nöthig zu haben, wer für ihre gesicherten Ergebnisse immer wieder glaubt fürchten zu müssen, thut ihrem Andenken schlechte Ehre an. Sehe ich recht, so ist Riehm in dies Verhalten erst hineingedrängt worden durch den passiven Widerstand gegen Fortschritte der Wissenschaft, denen er sich nicht zu beugen vermochte. Wie sehr dadurch ihm, den man sonst unter den Vorkämpfern unbefangener Schriftforschung zu finden gewohnt war, die Fühlung abhanden gekommen ist, kann man auch aus dẹr vorliegenden Recension ersehen. Denn es zeugt doch von auffallend geringem Verständnifs für Gesichtspunkte und Ziele der Gegner, dals Riehm es vom Standpunkte der Graf-Wellhausen'schen Hypothese aus für ein erzwungenes Zugeständnifs und eine beschönigte Niederlage ansehen kann, wenn immer mehr Punkte sich finden, in welchen die Grundschrift mit der jahwistischen Quelle übereinstimmt (S. 785). Wo es so sehr an der ersten Bedingung dazu fehlt, wird man leider die Hoffnung auf eine Verständigung noch weit hinausschieben müssen. Das endliche

1) Nur um solche handelt es sich hier, nicht um Veröffentlichungen für einen weiteren Leserkreis, akademische Vorträge oder dgl. 
Verdammungsurtheil, durch welches Riehm am Schlusse seiner Recension die theologische Wissenschaft vor kirchlicher Censurirung meint schützen zu sollen, dürfte bedeutend an Gewicht verlieren, wenn man sieht, wie er unbewufst und unbefangen dasselbe Verfahren, das er in dem Schlufssatze - allerdings in schwerlich zu billigender Schärfe der Fassung unter unberechtigter Berufung auf mein Buch S. 145 - rügt, in seiner Recension selbst mehrfach (z. B. S. 772 f. 778 f.) und in ziemlich ausgiebigem Umfạng handhabt. Oder bedingt das einen Unterschied, wenn er solche Vorgänge in die Verarbeitung der mündlichen Ueberlieferung verlegt, während ich aus ihnen die Zusammenschichtung der schriftlichen zu erklären suche? Mich dünkt, nur den, dafs ich eine höhere Schätzung der biblischen Schriftsteller vertrete, als Riehm, indem ich nicht ihnen selbst, sondern Redactorenhänden die Vereinigung widersprechender Ueberlieferungen zutraue.

\section{Philister und Hebräer zur Zeit Davids.}

\section{Von Adolf Kamphausen.}

Nicht um eine erschöpfende Darstellung des Verhältnisses, welches in der ältesten hebräischen Königszeit zwischen den Philistern und den Hebräern bestand, ist es mir jetzt zu thun, sondern ich möchte nur einige Bemerkungen mittheilen, welche vielleicht dies oder jenes in helleres geschichtliches Licht stellen helfen, zugleich aber einen Beitrag zu richtigerer Würdigung der Person Davids geben, den Ranke (Weltgesch. I, 1, S. 60. 63) als von Samuel gesalbten Propheten betrachtet, während der mit der neueren Kritik besser bekannte Ed. Meyer in seiner 